\title{
Improved survival rates in sebaceous carcinoma of the eyelid
}

MMK Muqit ${ }^{1}$, F Roberts ${ }^{2}$, WR Lee ${ }^{2}$ and E Kemp ${ }^{1}$

\begin{abstract}
Purpose To review the clinicopathological features, management, and survival rates for patients with sebaceous gland carcinoma of the eyelid, and to analyse the reasons for improved survival.

Methods In the west of Scotland between 1975 and 2001, 32 cases were identified through the pathology index for sebaceous carcinoma. Pathology specimens were reviewed in all cases. The clinical data were obtained from hospital records and the database provided by the Regional Cancer Registry.

Results A total of 22 female and 10 male patients were identified. Follow-up ranged from 6 months to 15 years, median of 4.8 years. Primary treatment involved excisional techniques in 27 cases, exenteration in two cases, enucleation in two cases, and two cases required adjuvant radiotherapy. Intraepithelial spread and masquerade presentations delayed the diagnosis. Poorly differentiated tumours were associated with an unfavourable outcome. Three patients developed local tumour recurrence and one metastatic disease. In all, 10 patients died from nontumour-related causes and one died from metastatic sebaceous carcinoma. The overall tumour mortality rate in the west of Scotland was $3 \%$.

Conclusions From this study, patients in the west of Scotland have a better prognosis than is indicated in the literature. Astute clinical suspicion and accurate histopathological diagnosis, together with radical and aggressive surgical approaches were key factors.

Eye (2004) 18, 49-53. doi:10.1038/sj.eye.6700523
\end{abstract}

Keywords: clinicopathological; eyelid; mortality; sebaceous gland carcinoma; surgical methods

\section{Introduction}

In the literature, mortality rates from primary sebaceous gland carcinoma vary from 9 to $40 \%,{ }^{1-6}$ and the 5-year mortality in patients with metastatic disease has been estimated at 50 $67 \% .^{5}$ Recent observations suggest that the prognosis for survival in patients with sebaceous gland carcinoma may be more favourable than the literature indicates. We reviewed archival details of patients $(1975-2001)$ in the west of Scotland. The aims of the study were to review the clinicopathological features, management, and survival rates in order to analyse the reasons for improved survival.

Materials and methods

A total of 32 cases were retrieved from the pathology index for sebaceous carcinoma. Conventional histology had been performed in conjunction with immunohistochemistry work described by Johnson et al, ${ }^{7}$ stains for fat and frozen sections. The clinical data including surgical management, recurrence rates, survival rates, and follow-up were obtained from general practice records, hospital records, and the database provided by the Regional Cancer Registry. The handling of all archival samples and the assessment of retrospective data were carried out in accordance with the Medical Research Council and General Medical Council ethical guidelines. Long-term follow-up data were only available for 22 cases owing to removal of medical records after the death (from other causes) of 10 patients.

Results

In total, 22 female (69\%) and 10 male (31\%) white Caucasians (ranging from 39 to 88 years) were included in this investigation. Characteristics of the study population are shown in Table 1. The left eye was involved in
${ }^{1}$ Tennent Institute of Ophthalmology Gartnavel General Hospital Glasgow, UK

${ }^{2}$ Department of Pathology University of Glasgow University Avenue Glasgow, UK

Correspondence: MMK Muqit Tennent Institute of Ophthalmology

Gartnavel general Hospital 1053 Great Western Road Glasgow G12 OYN, UK Tel: + 441412113000 E-mail:mmmk3@ aol.com

Received: 19 July 2002 Accepted in revised form: 24 January 2003

This work was presented at the Royal College of Ophthalmologists Annual Congress, May 2002 
Table 1 Characteristics of study population

\begin{tabular}{lcc}
\hline Patient factors & No. & $\%$ \\
\hline Sex & 10 & 31 \\
$\quad$ Male & 22 & 69 \\
Female & & \\
Age & $(39-88)$ & \\
$\quad$ Range & 67 & 53 \\
$\quad$ Mean & & 47 \\
Eye involved & 17 & \\
Right & 15 & 30 \\
Left & & 12.5 \\
Site & 16 & \\
UL & 12 & 45 \\
LL & 4 & 42 \\
UL and LL & & \\
Tumour size (mm) & & \\
0-10 & 14 & \\
11-20 & 13 & \\
21-50 & 4 & \\
Follow-up (months) & & \\
Range & & \\
Mean & $6-180$ & \\
\hline
\end{tabular}

15 cases and the right in 17 cases. In all, 16 cases were reported with delays in presentation from the onset to diagnosis, ranging from 1 month to 4 years (mean 15.8 months). The site of the primary lesion in 16 cases involved the upper lid, 12 cases the lower lid, and four cases involved both eyelids. The size of lesion (maximum diameter) in 31 cases varied from 2.5 to $50 \mathrm{~mm}$, with a mean as $13.5 \mathrm{~mm}$. We observed a higher recurrence rate for larger tumours ( $>13.5 \mathrm{~mm}$ ).

Clinical and pathological features are displayed in Figures 1 and 2. The most common initial misdiagnosis was squamous cell carcinoma (six cases), followed by basal cell carcinoma (five cases), meibomian cyst (three cases), chalazion (two cases), and single cases of papilloma, lymphoma, breast cancer metastasis, and blepharoconjunctivitis. However, the degree of clinical suspicion was very high in all cases except in one patient, which is discussed later. We analysed the specimens according to the presence of cytoplasmic vacuolation and foamy cytoplasm, the degree of differentiation, mitotic activity, amount of invasion/spread, and intraepithelial involvement. In total, 20 cases had typical features of pale cytoplasm and vacuolation, and 10 cases showed lobular growth patterns. In all, 13 cases showed poorly differentiated features, one moderately differentiated, and one of mixed cell type. There were three cases of intraepithelial spread, displaying intradermal and stromal invasion. This patient group had a favourable prognosis with no recurrences, which is contrary to the
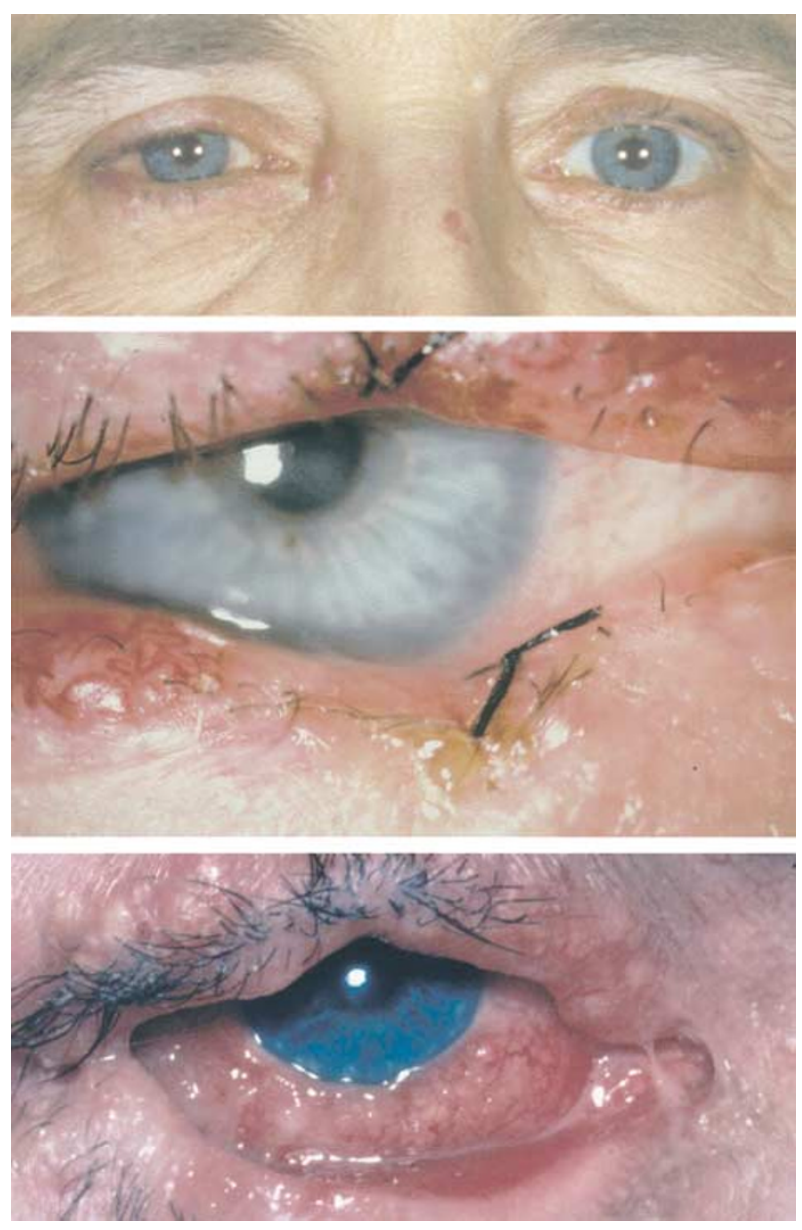

Figure 1 Upper: Unilateral blepharitis should arouse suspicion of a diffusely spreading sebaceous gland carcinoma. Middle: Biopsies were taken from the upper and lower lids (the histology is shown in Figure 2). Lower: Diagnosis was delayed in this patient who died from lung metastases after an orbital exenteration combined with a resection of the ipsilateral parotid gland and a neck dissection. The periocular dissemination of the sebaceous gland carcinoma is extensive.

literature. As reported, the presence of poorly differentiated tumour correlated with a poor prognosis, with three cases of local recurrence and one metastases. Signs of vascular invasion were only observed in the patient who developed metastatic disease.

\section{Management}

Primary treatment methods are shown in Table 2. Primary treatment involved wide local excision with $4 \mathrm{~mm}$ surgical margins in 19 cases, eight patients had wide local excision and eyelid reconstruction, one patient had local excision and radiotherapy, two cases of exenteration, one enucleation, and one case of enucleation and radiotherapy. In all, 28 patients $(87.5 \%)$ 

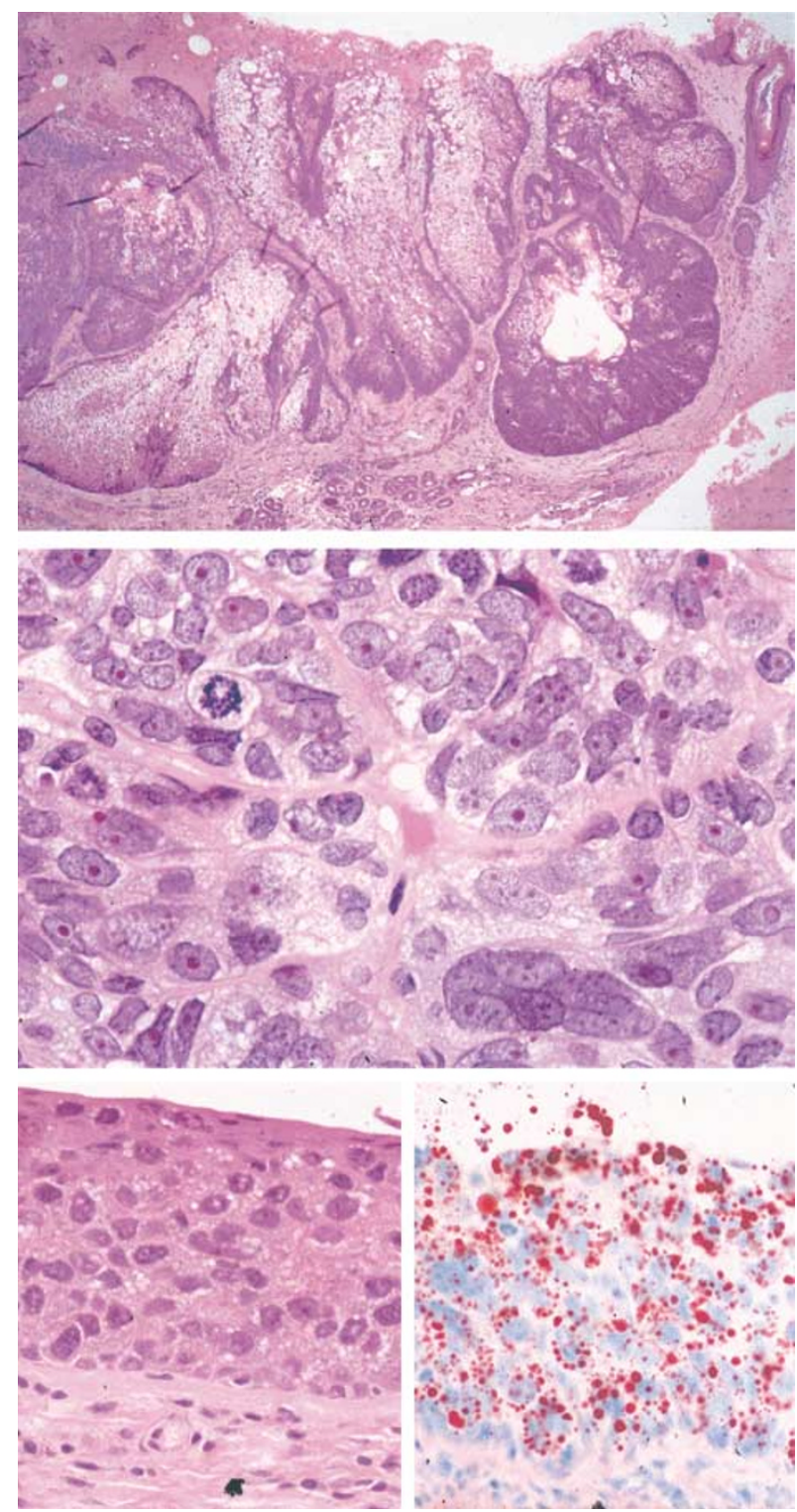

Figure 2 Upper: A well-differentiated sebaceous carcinoma resembles the structure of the meibomian gland. Middle: A poorly differentiated sebaceous carcinoma contains few cells with foamy cytoplasm (many cells do not possess features for classification). Lower: Conventional paraffin sections do not demonstrate the lipid-laden cells as well as those seen in a frozen stained with Oil red $\mathrm{O}$ for fat content. These specimens were taken from the Masquerade syndrome shown in Figure 1.

were successfully treated at the initial surgical intervention with zero recurrence.

A local recurrence of sebaceous gland carcinoma occurred in three patients (9.7\%). Two patients underwent secondary enucleation and the other required a wider re-excision plus Abbe switch reconstruction. The high clinical suspicion together with the early and aggressive surgical approaches, these factors significantly improved the outcome of this patient group. In one patient (Figure 1, lower), the diagnosis was delayed until parotid metastases brought him to the attention of the maxillofacial surgeons. This patient underwent a parotidectomy, an exenteration, block dissection of the neck and multiple grafts, but rapidly succumbed to lung metastases. The recurrence of sebaceous gland carcinoma following primary treatment was $9.7 \%$ for local recurrence and $3.1 \%$ for metastatic disease, with an overall rate of $12.5 \%$.

Follow-up periods ranged from 6 months to 15 years, involving regular annual reviews. A total of 15 patients had a minimum of 4-year follow-up, and the mean for the whole group was 4.8 years. There were eight deaths because of nontumour-related causes. According to the Cancer registry records, two patients died from unspecified, nontumour-related causes. None of the patients managed initially in ophthalmology departments died from metastatic disease.

\section{Discussion}

Sebaceous gland carcinoma of the eyelid is a relatively uncommon tumour that accounts for only 1 in 2000 of cutaneous malignancies, yet it is the second most common malignancy of the eyelid. ${ }^{8}$ It is slow growing and mimics several benign conditions, with the clinical appearances seldom pathognomonic. The most common masquerade syndrome proposed by various authors has been a chalazion..$^{9}$ Incorrect histopathological interpretations have been reported in $39-77 \%$ of cases. ${ }^{1,5,10}$

A delay of longer than 6 months between onset of symptoms and establishing the correct diagnosis has been noted to have a significantly adverse effect on patient mortality. ${ }^{2}$ Our findings correlate with published reports of the mean delay varying between 1 and 3 years. ${ }^{6}$ Most of these patients were referred from nonspecialised ophthalmic units. Clinical inexperience can result in a lower suspicion for atypical unilateral inflammatory eyelid pathology. The standard staining techniques routinely used by the hospital pathologists often produced equivocal results. There was usually a history of previous curettage or biopsies, with misdiagnosis. It is important to clearly demonstrate sebaceous differentiation within the group of eyelid neoplasms, which includes basal cell, squamous cell, and sebaceous gland carcinoma. Our ophthalmic pathologist provided a correct diagnosis on all the biopsies taken at the Tennent Institute of Ophthalmology.

It is important for the primary surgical intervention to clear the tissue of tumour, and we report a good outcome with surgical margins of $4 \mathrm{~mm}$. Adjuvant radiotherapy is considered palliative, ${ }^{3,11}$ and the decision to carry out 
Table 2 Results of primary surgical management

\begin{tabular}{|c|c|c|c|c|c|c|c|}
\hline Case & Age & Site & Size $(\mathrm{mm})$ & Recurrence & Management & Follow-up (months) & Cause of death \\
\hline $1^{\mathrm{a}}$ & 47 & LLL & 8 & & & 180 & \\
\hline $2^{a}$ & 56 & RLL & 15 & & & 24 & \\
\hline $3^{\mathrm{a}}$ & 59 & RUL & 2.5 & & & 72 & \\
\hline $4^{\mathrm{a}}$ & 56 & RUL & 20 & Local & Enucleation & 96 & Lung CA \\
\hline 5 & 74 & LUL & 14 & & & 48 & MI \\
\hline 6 & 63 & RLL & 13 & & & 48 & \\
\hline 7 & 63 & LUL & 9 & & & 12 & \\
\hline 8 & 60 & LUL/LL & 5 & & & 60 & \\
\hline 9 & 77 & RUL/LL & 10 & & & 12 & Unspecified \\
\hline $10^{\mathrm{b}}$ & 40 & LUL/LL & 30 & & & 84 & \\
\hline 11 & 39 & LUL & 5 & & & 12 & \\
\hline 12 & 61 & RUL & 3 & & & 156 & \\
\hline $13^{c}$ & 65 & LUL & 10 & & & 12 & Stroke \\
\hline 14 & 77 & LUL & 17 & & & 12 & \\
\hline $15^{\mathrm{a}}$ & 77 & LUL & 5 & & & 156 & \\
\hline 16 & 76 & LLL & 9 & & & 12 & Peptic ulcer \\
\hline 17 & 65 & LUL & 9 & & & 90 & \\
\hline $18^{\mathrm{a}}$ & 83 & RLL & 19 & & & 12 & Prostate CA \\
\hline $19^{a}$ & 75 & RUL & 12 & & & 24 & IHD \\
\hline 20 & 70 & RLL & 20 & Local & Enucleation & 8 & \\
\hline $21^{\mathrm{d}}$ & 88 & LUL & 20 & & & 12 & Unspecified \\
\hline 22 & 45 & LLL & 2.5 & & & 120 & \\
\hline $23^{e}$ & 74 & LUL & 30 & & & 120 & \\
\hline 24 & 44 & RLL & 1 & & & 120 & \\
\hline 25 & 77 & RLL & 12 & & & 96 & \\
\hline 26 & 75 & RUL & 15 & & & 41 & \\
\hline $27^{\mathrm{a}}$ & 79 & LUL & 50 & & & 9 & \\
\hline 28 & 71 & LUL & 10 & & & 6 & \\
\hline 29 & 73 & LUL & 20 & Local & WLE $x 2$ & 72 & \\
\hline 30 & 83 & RLL & 10 & & & 84 & Emphysema \\
\hline 31 & 84 & RUL & 14 & & & 12 & Stroke \\
\hline $32^{\mathrm{d}}$ & 50 & RUL/LL & NA & Lymphatic & $\mathrm{P} / \mathrm{LND} / \mathrm{R}$ & 10 & Sebaceous CA \\
\hline
\end{tabular}

${ }^{a}$ WLE and reconstruction. ${ }^{b}$ Enucleation and radiotherapy. ${ }^{c}$ WLE and radiotherapy. ${ }^{\mathrm{d} E x e n t e r a t i o n . ~}{ }^{\mathrm{e} E n u c l e a t i o n . ~ R U L / L L=r i g h t ~ u p p e r ~ l i d / l o w e r ~ l i d, ~}$ LUL/LL=left upper lid/lower lid, IHD=ischaemic heart disease, MI=myocardial infarction, CA=carcinoma, P/LND/R=parotidectomy, lymph node dissection, reconstruction with grafts.

Table 3 Review of survival rates in the literature

\begin{tabular}{lrcc}
\hline Author & $\begin{array}{c}\text { Cases } \\
(\text { no. })\end{array}$ & $\begin{array}{c}\text { Mortality rate } \\
(\%)\end{array}$ & $\begin{array}{c}\text { Follow-up } \\
\text { (years) }\end{array}$ \\
\hline Muqit et al (2002) & 32 & 3 & 5 \\
Zurcher et al (1998) & 43 & 9 & 3 \\
Doxanas et al (1984) & 40 & 15 & NA \\
Rao et al (1982) & 104 & 22 & 5 \\
Ni et al (1982) & 82 & 24 & 4 \\
Ni and Kou (1979) & 100 & 41 & $5-15$ \\
Boniuk et al (1968) & 88 & 30 & 5 \\
\hline
\end{tabular}

enucleation and exenteration procedures must be justified since such surgery can be mutilating. In our study, large advanced tumours and/or orbital infiltration would not allow the surgeon to take adequate surgical margins, so exenteration was carried out with good results.

This study highlights the importance of astute clinical suspicion, early diagnosis, accurate histopathological diagnosis, aggressive primary and secondary surgical techniques, and long follow-up. The mortality from sebaceous carcinoma of the eyelid has been estimated to be from 9 to $40 \%^{1-6}$ (see Table 3). Although 10 of our 32 patients died over the 26-year period of study, only a single case was registered as death caused by metastatic sebaceous gland carcinoma. In the west of Scotland, patients with sebaceous gland carcinoma of the eyelid have encountered a much better prognosis than that previously reported in the literature.

\section{References}

1 Zurcher M, Hintschich, Garner A, Bunce C, O Collin JR. Sebaceous carcinoma of the eyelid: a clinicopathological study. Br J Ophthalmol 1998; 82: 1049-1055.

2 Doxanas MT, Green WR. Sebaceous gland carcinoma: review of 40 cases. Arch Ophthalmol 1984; 102: 245-249. 
3 Rao NA, Hidayat AA, McLean JW, Zimmerman LE. Sebaceous carcinoma of the ocular adnexa. Hum Pathol 1982; 13: 113-122.

4 Ni C, Searle SS, Kuo PK. Tumours of the eyelid and orbit: A Chinese-American Collaborative Study. Int Ophthalmol Clin 1982; 22: 23-61.

5 Ni C, Kuo PK. Meibomian gland carcinoma: a clinicopathologic study of 156 cases with long-period follow-up of 100 cases. Jpn J Ophthalmol 1979; 23: 388-401.

6 Boniuk M, Zimmerman LE. Sebaceous carcinoma of the eyelid, eyebrow, caruncle and orbit. Trans Am Acad Ophthalmol Otolaryngol 1968; 72: 619-641.

7 Johnson JS, Lee JA, Cotton DWK, Lee WR, Parsons MA. Dimorphic immunohistochemical staining in ocular sebaceous neoplasms: a useful diagnostic aid. Eye 1999; 13: 104-108.

8 McLean IW, Jackobiec FA, Zimmerman LE, Burnier MM. Tumours of the Eye and Ocular Adnexa, Vol 3. Maryland Armed Forces Institute of Pathology: Washington, 1993, pp 28-35.

9 Nelson BR, Hamlet KR, Gillard M, Railan D, Johnson TM. Sebaceous carcinoma. J Am Acad Dermatol 1995; 33: 1-15.

10 Yeatts RD, Waller RR. Sebaceous carcinoma of the eyelids: pitfalls in diagnosis. Ophthalmic Plast Reconstr Surg 1985; 1: 35-42.

11 Nunery WR, Welsh MG, McCord CD. Recurrence of sebaceous carcinoma of the eyelid after radiation therapy. Am J Ophthalmol 1983; 96: 10-15. 